RESEARCH NOTE

\section{Virulent and Avirulent Strains of Group B Streptococci from Rio de Janeiro, Brazil. Relationship between Differences in Surface Hydrophobicity, Sialic Acid Content and Macrophage Interaction}

\section{Prescilla E Nagao $/{ }^{+}$, Leslie C Benchetrit*}

Departamento de Biologia Celular e Genética, Instituto de Biologia Roberto Alcântara Gomes, Universidade do Estado do Rio de Janeiro, Rua São Francisco Xavier 524, PHLC, sala 205, 20550-013 Rio de Janeiro, RJ, Brasil *Instituto de Microbiologia Prof. Paulo de Góes, Universidade Federal do Rio de Janeiro, Rio de Janeiro, RJ, Brasil

Key words: streptococci - surface hydrophobicity sialic acid - macrophages - Rio de Janeiro

Bacterial attachment to epithelia is an important mechanism for colonization of mucosal membranes and influences microbial pathogenicity. A number of studies with both Gram-positive and Gram-negative bacteria has indicated that this hostmicro-organism interaction is determined by different factors. The degree of hydrophobicity of the bacterial can influence cell adhesion (GI Geertsema-Doornbusch et al. 1993 J Microbiol Methods 18: 61-68, P Gilbert et al. 1993 J Appl Bacteriol (Suppl.) 74: 67S-78S) and phagocytosis (CJ van Oss 1978 Ann Rev Microbiol 32: 19-39).

The streptococci of group B (GBS) are important pathogens involved in human and animal diseases (JL Nancy et al. 1984 J Infect Dis 149: 851860, GS Tamura et al. 1994 Infect Immun 62: 24502458, P Munoz 1997 Arch Intern Med 157: 213-

\footnotetext{
This work was supported by $\mathrm{CNPq}$ and Faperj.

${ }^{+}$Corresponding author. Fax: +55-21-587.7377. E-mail: pnagao@uerj.br

Received 11 November 1998

Accepted 2 March 1999
}

216). A wide variety of infections has been described ranging from minor lesions to disseminated diseases. Because hydrophobicity seems to be an important factor that can influence bacterial-eucaryotic cell interactions, in the study presented here we examined the cell surface hydrophobicity of GBS from different clinical sources and examined the role of this surface property on the GBSmacrophage interaction.

Two strains of GBS serotype III were used in this study. The strain 80340 was collected from the urethra of an asymptomatic patient and the other strain (90356) from a patient presenting meningitis. Bacteria were stored in the lyophilized state and recovered, when necessary, in broth (Brain Heart Infusion, Difco) supplemented with 5\% sheep blood.

For the hydrophobicity assay, single (three) colonies were picked from sheep blood plates and grown for $18 \mathrm{hr}$ at $37^{\circ} \mathrm{C}$. The bacteria were then harvested by centrifugation, washed twice and suspended in $5 \mathrm{ml}$ of $0.05 \mathrm{M}$ sodium phosphate buffer (pH 7.4) containing $0.15 \mathrm{M} \mathrm{NaCl}$ to an absorbency of 0.4 at $660 \mathrm{~nm}$. The bacterial suspension was treated with xylene $(2.5: 1, \mathrm{v} / \mathrm{v}$; Merck), the system was vigorously mixed for $2 \mathrm{~min}$ and allowed to settle for $20 \mathrm{~min}$ at room temperature. The absorbency of the aqueous phase was then determined at $660 \mathrm{~nm}$ against a blank consisting of sodium phosphate buffer. The hydrophobicity indexes (HI) were calculated as previously described (M Rosenberg et al. 1980 FEMS Microbiol Lett 9: 2933).

Peritoneal macrophages were collected from Swiss mice and allowed to adhere to coverslips as previously described ( $\mathrm{P}$ Valentin-weigand et al. 1996 Infect Immun 64: 2467-2473). The bacteriamacrophage ratio was adjusted to 10:1 and interactions were allowed to proceed for periods of 15 min to $240 \mathrm{~min}$. After interaction, adherent cells were washed, fixed in Bouin for 5 min, stained with Giemsa, and the mean number of attached microrganisms per infected macrophage was determined by counting the number of adherent bacteria in 900 phagocytes by light microscopy.

All the procedures for extraction, purification and determination of sialic acid content of GBS were carried out as formally described (JP Kamerling et al. 1982 Biochem Biophys 714: 351354).

The bacteria-macrophage ratios were analyzed by the Kolmogorov-Smirnov test using the "Software Statistica" version 5.0 "Release" 5.1 (S Siegel 1977, Estatística Não Paramétrica, McGraw-Hill, São Paulo, 350 pp.). P $\leq 0.05$ was considered statistically significant. 
Our results showed that a statistically significant difference was observed for the GBS-macrophage interaction between the more and less hydrophobic GBS strains. The more hydrophobic strain from GBS serotype III (strain 80340) presented a higher macrophage interaction ratio (Fig., Table; $\mathrm{P}<0.01)$ when compared with that of the less hydrophobic GBS isolate (90356). The highest degree of adhesion was observed after $60 \mathrm{~min}$ of bacteria-macrophage contact. Thereafter, the ratio was lower for all of the samples analyzed (Fig.).

Since the capsular polysaccharide of type III GBS contributes substantially to the virulence of this organism (HH Yim et al. 1997 Adv Exp Med Biol 418: 995-997) and also significantly influences the surface HI, we explored the extent to which the content of sialic acid residues in these clinical isolates would affect the GBS-macrophage interactions. We verified that the less interactive and less hydrophobic type III GBS strain presented an increased sialic acid content (almost 100\% higher) when compared with that of the more hydrophobic strain 80340 (Table). It is interesting to observe that the strain 90356, that presented a very low macrophage interaction ratio, and also presented a high sialic acid content concomitantly with lower

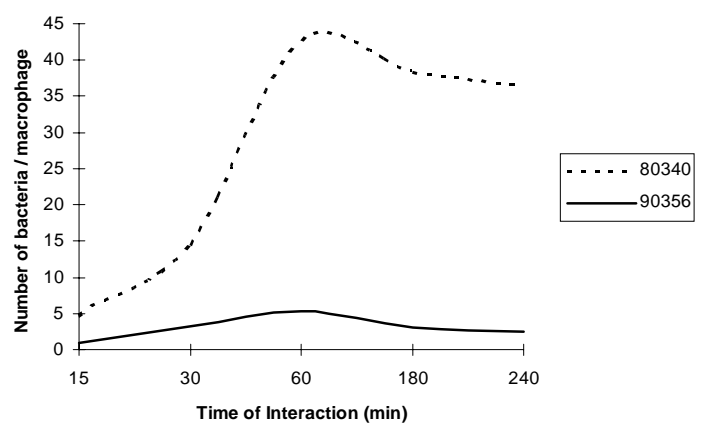

Attachment of group B streptococci serotype III to macrophages.
$\mathrm{HI}$, was an isolate that was causing meningitis in a newborn human. These data imply that the difference observed in the pattern of GBS type III-macrophage interactions was probably due to the difference on the composition of GBS type III cell surface that resulted in a lower HI.

The adhesion mechanism is very complicated, and different adhesive properties of the bacteria are involved, including hydrophobic interaction, protein-protein interaction, and protein-carbohydrate interaction, including sialic dependent lectins.

Sialic acid content seems to contribute to the difference observed in the cell surface HI of GBS clinical isolates. Although the molecular basis of these interactions is not well-understood, it is possible that in the absence of specific immunity in neonates, the very low macrophage interaction pattern observed for the less hydrophobic and highlevel sialic acid-content type III isolate represents a significant virulence determinant for GBS type III invasive isolates, which poorly activate the alternative complement pathway. Although we did not explore this possibility, one can not rule out the hypothesis that proteinaceous structures that may contribute for GBS HI would also be involved in the type III resistance to non-opsonic phagocytosis of some GBS isolates. We are now developing studies to characterize the macrophage receptors and the GBS surface structures involved in these interactions, to determine how GBS type III polysaccharide of an invasive clinical isolate can specifically regulate GBS interactions with macrophage receptors.

Additional studies will be required to identify the bacterial residues that mediate surface hydrophobicity and the mode of attachment of these molecules on GBS cell surface. Finally, a very important aspect will be the elucidation of the exact molecular basis for protein-carbohydrate interaction in order to further clarify bacterial-host cell physical interactions.

TABLE

Surface hydrophobicity and sialic acid content of group B streptococci from different clinical sources

\begin{tabular}{lcccc}
\hline Strains & Serotype & Clinical origin & Hydrophobicity index & $\begin{array}{c}\text { Sialic acid } \\
\mu \mathrm{g} / \mathrm{mg} \text { bacterial cell }\end{array}$ \\
\hline 80340 & III & Urethra & 82.1 & 4.96 \\
90356 & III & Cerebrospiral fluid & 42.7 & 8.79 \\
\hline
\end{tabular}

